\title{
Steady flows of a laterally heated ferrofluid layer: Influence of inclined strong magnetic field and gravity level
}

\author{
M. Hennenberg \\ Microgravity Research Center, Chimie-Physique Ecole Polytechnique Faculty of Applied Sciences, \\ Code Postal 165/62, Université Libre de Bruxelles, Av. F.D. Roosevelt 50, Brussels B-1050, Belgium
}

\begin{abstract}
B. Weyssow
Association Euratom-Etat Belge, Faculty of Sciences, Code Postal 231, Université Libre de Bruxelles, Blvrd du Triomphe, Brussels B-1050, Belgium

S. Slavtchev

Institute of Mechanics, Bulgarian Academy of Sciences, Acad. G. Bontchev Str., Block 4, Sofia 1113, Bulgaria

Th. Desaive

Institute of Physics, University of Liège, Allée du 6 Aôut 17, B5, B-4000 Liège, Belgium

B. Scheid

Microgravity Research Center, Chimie-Physique Ecole Polytechnique Faculty of Applied Sciences, Code Postal 165/62, Université Libre de Bruxelles, Av. F.D. Roosevelt 50, Brussels B-1050, Belgium
\end{abstract}

(Received 10 August 2006; accepted 15 August 2006; published online 20 September 2006)

\begin{abstract}
A horizontal ferrofluid layer is submitted to a lateral heating and to a strong oblique magnetic field. The problem, combining the momentum and heat balance equations with the Maxwell equations, introduces two Rayleigh numbers, $\mathrm{Ra}$ the gravitational one and $\mathrm{Ra}_{m}$ the magnetic one, to represent the buoyancy and the Kelvin forces, which induce motion, versus the momentum viscous diffusion and heat diffusion. Whatever the inclination of the magnetic field, the steady solution of the problem is presented as a power series of a small parameter $\epsilon_{H}$ measuring the ratio of variation of the magnetization across the layer divided by the magnitude of the external imposed field. For cases of physical relevance, comparisons between analytical and numerical studies have lead to a major statement: in the strong field region $\left(\epsilon_{H} \ll 1\right)$ the zero order solution is the product of the Birikh solution that corresponds to the usual Newtonian fluid submitted to a lateral gradient, multiplied by a modulating factor accounting for inclination and both Rayleigh numbers. Physically, this simplified solution is valid for microgravity conditions where the magnetic field competes enough with microgravity effects to invert the laminar flow and thus suppress the motion for two specific values of the inclination angle. (C) 2006 American Institute of Physics. [DOI: 10.1063/1.2353879]
\end{abstract}

\section{INTRODUCTION}

The Rayleigh-Bénard problem has become a paradigm of nonlinear stability studies, since the famous book of Chandrasekhar. ${ }^{1}$ The thermoconvective problem induced by a lateral gradient of temperature is also a well-studied problem dating back to the original works of Birikh, ${ }^{2}$ or Kirdyashkin ${ }^{3}$ and Davis. ${ }^{4}$ The initial Rayleigh-Bénard problem linked to a temperature gradient opposed to gravity and the one, induced by a lateral gradient of temperature, ${ }^{2,4}$ can be extended to a ferrofluid taking also into account an externally imposed magnetic field. When such a fluid is submitted to a gradient of temperature, the momentum balance experiences a profound modification, through the Kelvin force reflecting the magnetization of the ferrofluid. From a theoretical point of view, the stability studies of a temperature gradient normal to the horizontal boundaries, are giving rise to new perspectives for those kinds of fluids, as is well known since the classical works of Finlayson, ${ }^{5}$ Neuringer and Rosensweig, ${ }^{6}$ and Bashtovoi and Berkovski. ${ }^{7}$ Indeed, a ferrofluid, submitted to a vertical magnetic field parallel or antiparallel to gravity, can give rise to a nonoscillatory insta- bility that appears whether one heats from above or from below, ${ }^{8}$ under very precise critical conditions.

We will consider the layer of ferrofluid to be of infinite lateral extent and to be sandwiched between two rigid plates and to be submitted to an inclined strong magnetic field so that the magnetization of the liquid is only temperature dependent (see Fig. 1). This however does not apply to thermocapillarity. Indeed, the present formulation, does not apply to a free surface configuration, ${ }^{9}$ since the external applied field $H^{e}$ is larger than the critical field giving rise to the static Cowley-Rosensweig change of shape of a ferrofluid layer adjacent to an inert gas or another liquid. ${ }^{8-12}$ For a large layer of an isothermal ferrofluid, this nonoscillating instability appears for any field stronger than the critical magnetic field $H_{\text {crit }}$ defined by

$$
H_{\text {crit }}=\sqrt{\frac{2}{\mu_{0}} \frac{1+\mu_{0} / \mu}{\left[\mu / \mu_{0}-1\right]^{2}} \sqrt{\rho \sigma g}} \text { for } k_{\text {crit }}=\sqrt{\frac{\rho g}{\sigma}},
$$

where $\rho$ is the density of the ferrofluid, $\mu\left(\mu_{0}\right)$ the magnetic permittivity of the ferrofluid (respectively, of the void), $g$ the gravity and $\sigma$ the surface tension. This instability is due to 


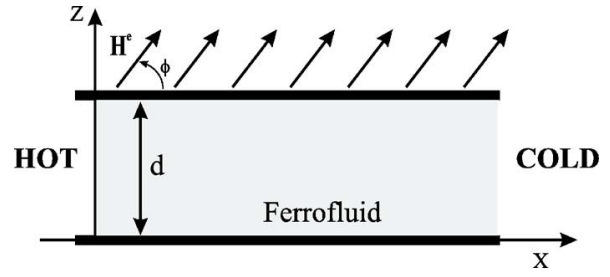

FIG. 1. Laterally heated layer of ferrofluid submitted to an inclined magnetic field $H^{e}$.

the balance between the magnetic traction, the Laplace pressure and gravity along a free ferrofluid interface. Let us note that $H_{\text {crit }}$ varies as $g^{1 / 4}$ so that it will fall down to very low values in microgravity environment. Thus, a reference free surface cannot remain flat for a strong magnetic field. Even if we do not study stability at the present stage, we should keep in mind this specific property of ferrofluid.

In Sec. II, we present the formalism of our problem and in Sec. III the adimensionalization, while in Sec. IV we discuss the inductive assumption. Section V introduces the final form of the dimensionless balance equations and gives the temperature and velocity profiles. To obtain these analytical solutions, we introduced some physically simplifying hypothesis discussed in Sec. VI, using a well defined ferrofluid EMG 901. Then we discuss in Sec. VII, the profiles in terms of the intervening dimensionless parameters of the magnetic field inclination and strength as well as the gravity level, using as a benchmark the classical Birikh solution. ${ }^{2-4}$ In that paragraph, we show that our approximation should lead for a low gravity level and very precise inclinations to a heat conductive motionless case, so that one could observe a reverse of the flow and of the temperature profiles for an inclined magnetic field whose inclination varies between those critical values. We finally compare in Sec. VIII our solution to an exact analytical solution and discuss the validity range in term of the parameter $\epsilon_{H}$. We summarize our main results in the conclusion (see Sec. IX).

\section{FORMULATION OF THE PROBLEM}

We want to get an analytic form for the steady state of a ferrofluid submitted to the lateral heating and to an inclined magnetic field, to serve as a reference state for a future stability investigation. As we limit ourselves to a $2 \mathrm{D}$ description, we are in fact considering the central region of a very elongated and thin layer, so that we forget the influence of the lateral walls, supposed to be very far from the described region. Still we must suppose that the temperature at the hottest lateral wall is below the boiling one and that the temperature along the coldest lateral wall is above the freezing one. These precautions are mandatory to design a set of parameters which are not only numerically, but also physically reasonable.

Let a ferrofluid layer of width $d$ between two horizontal rigid plates, be submitted to an inclined magnetic field and to a lateral gradient of temperature (see Fig. 1). We combine thus the initial 2D situation considered for Newtonian fluids ${ }^{2,4}$ to the one taking into account the specific properties of a ferrofluid. ${ }^{7,9}$ We will show that, in the limit of a strong magnetic field, the steady reference regime state can be described by a set of two-dimensional balance laws. The reference regime state shows some new possibilities which deserve a careful study and should be tested experimentally on Earth and in microgravity.

\section{A. The ferrofluid magnetization and the Maxwell equations}

To this layer of ferrofluid, we apply an exterior constant magnetic field, given by

$$
\mathbf{H}^{e}=H^{e}\left(\cos \phi \mathbf{1}_{x}+\sin \phi \mathbf{1}_{z}\right)=H^{e} \mathbf{1}_{H},
$$

where $\mathbf{1}_{z}$ is the upward unit normal, $\mathbf{1}_{x}$ is the horizontal unit taken along the lower border and $\mathbf{1}_{H}=\mathbf{H} / \sqrt{\mathbf{H} \cdot \mathbf{H}}$ (see Fig. 1). The ferrofluid is sufficiently dilute to be assimilated to a super paramagnetic gas, ${ }^{10}$ so that the magnetization is described as $M=n m \mathcal{L}(\xi)$ where the Langevin function $\mathcal{L}(\xi)$ is defined by $\mathcal{L}(\xi)=\operatorname{coth} \xi-\frac{1}{\xi}$ (Ref. 10) with the quantity $\xi$ $=\mu_{0} \mathrm{mH} / \mathrm{kT}, n$ is the number of nanomagnets per unit of continuum volume, $m$ the magnetic moment of one particle, $H$ the local value of the applied field, $k T$ the Brownian energy of chaotic motion ( $k$ being the Boltzmann constant) and $T$ the temperature.

Since $H^{e}$ is time independent, we neglect the Brownian and the Néel relaxation mechanisms. ${ }^{7,10}$ Therefore, we suppose that the magnetization is collinear with the magnetic field

$$
\mathbf{M}=M \mathbf{1}_{H} .
$$

For very strong fields, where $\xi \gg 1$, the magnetization is equal to its saturation value and does no more depend on the magnetic field. ${ }^{7,8}$ This situation corresponds to a very large sample of practical cases, so that we will assume that

$$
\frac{M(T)}{H}=O\left(\epsilon_{H}\right) \quad \text { where } \epsilon_{H} \ll 1 \forall x, z .
$$

We will explicitly define $\epsilon_{H}$ below and use the consequences of that approximation ${ }^{7,10}$ to obtain the following linearized form of the magnetization state equation $M=M(T)$,

$$
M=M\left(T_{0}, H_{0}\right)+\left(\frac{\partial M}{\partial T}\right)_{H_{0}}\left(T-T_{0}\right),
$$

near the reference state given by $M\left(T_{0}, H_{0}\right)$. Equation (4a) introduces the pyromagnetic coefficient $K=-(\partial M / \partial T)_{H_{0}}$ which, for large fields, ${ }^{8}$ is assumed to be constant and is given by

$$
K=\alpha M\left(T_{0}\right),
$$

where $\alpha=-\rho^{-1} \partial \rho / \partial T$ is the thermal expansion coefficient.

In the ferrofluid layer, ${ }^{5,7,8,10}$ there is no electrical current, so that the Maxwell equations reduce to

$$
\boldsymbol{\nabla} \times \mathbf{H}=0, \quad \boldsymbol{\nabla} \cdot[\mathbf{H}+\mathbf{M}]=\nabla \cdot\left[\left(1+\frac{M}{H}\right) \mathbf{H}\right]=0 .
$$

The second equation (5a) should be further expanded since, from (4a), the magnetization $M$ depends upon the temperature $T$, and it finally reads 


$$
\boldsymbol{\nabla} \cdot \mathbf{H}=\alpha M(T) \mathbf{1}_{H} \cdot \nabla T=K \mathbf{1}_{H} \cdot \nabla T .
$$

We will take into account the influence of the right-hand side of the last equation. As this term is different from zero, we explicitly link the magnetic inclined field $\mathbf{H}$, to the lateral gradient of temperature $T$. We assume thus that what is called the inductive assumption applies here. ${ }^{8}$

\section{B. The basic momentum balance and the return flow assumption}

We describe here the reference steady motion as a consequence of a basic assumption: in the reference state, exists a horizontal flow, whose velocity can be developed in a power expansion of $\epsilon_{H}$ with its zeroth order term depending on the $z$ coordinate only. Thus, one has

$$
\mathbf{u}=\left[u(z)+O\left(\epsilon_{H}\right)\right] \mathbf{1}_{x} .
$$

The problem now is to find explicitly the function $u(z)$. This simply extends to the present ferrofluid problem, the results of former scientists ${ }^{2-4}$ who studied pure Newtonian fluid layers submitted to a lateral heating.

As a consequence of the ferrofluid incompressibility and of the fundamental assumption (6a), there is no net mass flux across a vertical section of the ferrofluid layer. It means that

$$
\int_{0}^{d} u(z) \mathrm{d} z=0
$$

The Boussinesq approximation of the momentum balance equation is then ${ }^{1,5,10}$

$$
0=-\nabla p+\mu_{0} M \nabla H+\eta \frac{d^{2} u}{d z^{2}} \mathbf{1}_{x}-\rho_{0} g\left[1-\alpha\left(T-T_{0}\right)\right] \mathbf{1}_{z},
$$

where $\rho_{0}$ is the density at the reference temperature $T_{0}$ and $\eta$ is the kinematic viscosity. We neglect thermomagnetophoresis. $^{13-15}$

Eliminating the pressure from (6c), one obtains immediately

$$
0=\eta \frac{d^{3} u}{d z^{3}}-\alpha \rho_{0} g \frac{\partial T}{\partial x}+K \mu_{0}\left(\frac{\partial T}{\partial x} \frac{\partial H}{\partial z}-\frac{\partial T}{\partial z} \frac{\partial H}{\partial x}\right) .
$$

To be coherent with our basic assumption (6a), we must have

$$
\alpha \rho_{0} g \frac{\partial T}{\partial x}-K \mu_{0}\left(\frac{\partial T}{\partial x} \frac{\partial H}{\partial z}-\frac{\partial T}{\partial z} \frac{\partial H}{\partial x}\right)=G(z),
$$

so that all partial derivatives $\partial T / \partial x, \partial T / \partial z, \partial H / \partial x$ and $\partial H / \partial z$ should be functions of $z$ or constants.

\section{The energy equation}

The energy balance equation for the ferrofluid is the one derived by Finlayson, ${ }^{5}$ given by

$$
\rho c_{p} u \frac{\partial T}{\partial x}-\mu_{0} K u \frac{\partial H}{\partial x}=\lambda \nabla^{2} T,
$$

where $c_{p}$ is the heat capacity of the ferrofluid and $\lambda$ is the thermal conductivity. As usual for this kind of problems, ${ }^{5,7,8,10}$ we will neglect the advective magnetic term $\mu_{0} K u \frac{\partial H}{\partial x}$ so that the energy equation is the usual Fourier equation

$$
u \frac{\partial T}{\partial x}=\kappa \nabla^{2} T
$$

where $\kappa=\lambda / \rho c_{p}$ is the heat diffusion coefficient.

\section{The boundary conditions}

The above set of equations is solved using the following boundary conditions:

(a) Both the lower and upper borders are rigid nonmagnetic plates and the ferrofluid is a viscous liquid, adhering to a solid boundary, so that

$u(z)=0 \quad$ at $z=0, d$.

(b) The normal component of the magnetic induction $\mathbf{B}$ $=\mu_{0}[\mathbf{H}+\mathbf{M}]$ and the tangential component of the magnetic field $\mathbf{H}$ have to be continuous across the top and the bottom boundaries: $:^{5,10,13,16,17}$

$H_{z}+M_{z}=H^{e} \sin \phi, \quad$ and $\quad H_{x}=H^{e} \cos \phi$.

(c) All along both horizontal boundaries of the ferrofluid layer, whatever the $x$ coordinate, the lateral gradient of temperature is given by

$\frac{\partial T}{\partial x}=-\beta, \quad$ where $\beta>0$,

so that the temperature along the ferrofluid lateral boundaries is $T_{\text {ref }}-\beta x$, where $T_{\text {ref }}$ is the temperature at $x=0$. This simple condition was initially used by Birikh, ${ }^{2}$ but it could be easily removed. Here, the temperature decreases when the horizontal coordinate $x$ increases. Physically, $\beta$ is the absolute value of the variation of temperature per unit length.

\section{DIMENSIONLESS FORMULATION}

Let us rewrite the complete problem in a dimensionless form: the temperature is scaled by $\beta d$ and the velocity by $\kappa / d$. We are using $d$ as reference length, to scale both spatial coordinates $x$ and $z$, so that henceforward, keeping the same notations we have $0 \leq z \leq 1$. The magnetic field $\mathbf{H}$ and the magnetization $\mathbf{M}$ are scaled by $H^{e}$ which is the externally applied magnitude of the magnetic fluid.

The dimensionless form of (4a) is

$$
M=M_{0}-\epsilon_{H}\left(T-T_{0}\right),
$$

which introduces the quantity $\epsilon_{H}=K \beta d / H^{e}$. Due to our basic assumption (3), $\epsilon_{H}$ is a very small parameter since it is the scaling of the variation of the magnetization.

We will keep the same notation for the physically dimensionless quantities and continue our discussion only in terms of these new variables. Using (8b) and (8c), a solution of (6d), that fulfills the requirement imposed by Eq. (6e), will be

$$
T=T_{\text {ref }}-(x+t(z)),
$$




$$
H=\alpha_{1} x+h(z),
$$

where $t(z)$ and $h(z)$ are dimensionless functions of $z$. From (8c), one has $t(0)=0$ as well as $t(1)=0$, which physically means that the temperature along both horizontal borders varies only with the horizontal coordinate.

Since $H$ is a linear function of $x$, we will suppose that each component is a linear function of $x$

$$
H_{x}=A(z) x+h_{x}(z) \quad \text { and } \quad H_{z}=A_{1}(z) x+h_{z}(z) .
$$

It follows from (8b) that we must have $A=0$ at $z=0,1$ and $h_{x}(z)=\cos \phi+g(z)$ with

$$
g(0)=g(1)=0 .
$$

To obey (6e), $\alpha_{1}$ must be a constant, which we will show to be of order $\epsilon_{H}$, to avoid having $\partial H / \partial z$ as a function of $x$. Accordingly, $A_{1}(z)$ and $A(z)$ are also of $\mathrm{O}\left(\epsilon_{H}\right)$, which insure small variations of the magnetic field along the $x$ coordinate. We will develop the dimensionless unknowns, the velocity $u(z)$, the temperature $t(z)$, the norm of the magnetic field $H$, and its components $H_{x}$ and $H_{z}$, the magnetization $M$ as well as $h(z), h_{x}(z), h_{z}(z)$ in a power expansion of $\epsilon_{H}$, up to the first order in $\epsilon_{H}$, so that one has

$$
q(z)=q_{0}(z)+\epsilon_{H} q_{1}(z)+O\left(\epsilon_{H}^{2}\right),
$$

where $q(z)$ represents any of the variables.

\section{THE MAGNETIC FIELD AND THE INDUCTIVE APPROXIMATION}

Now from the Maxwell equation $\nabla \times \mathbf{H}=\mathbf{0}$, using (11)

$$
A_{1}=\frac{\partial H_{z}}{\partial x}=x \frac{d A}{d z}+\frac{d g}{d z}=\frac{\partial H_{x}}{\partial z} .
$$

But, since $A_{1}$ does not depend upon $x$, Eq. (14a) is only possible if $A$ is a constant, so that $d g(z) / d z=A_{1}$ and thus, using (12), $g(z)$ must be of $O\left(\epsilon_{h}\right)$. Let us write

$$
g(z)=\epsilon_{H} g_{1}(z) .
$$

Thus, across the whole layer $0 \leq z \leq 1, A=0$ and $H_{x}$ is independent of $x$. This result is a consequence of the approximation for the velocity, i.e., $\mathbf{u}=u(z) \mathbf{1}_{x}$. The only dependence upon $x$ of the magnitude of the field $H$ is proportional to $\alpha_{1}$.

\section{A. Consequence of the Maxwell boundary conditions at $z=0,1$}

The dimensionless expression of the collinearity (2) is

$$
\begin{aligned}
& \mathbf{M}(T)=M(T) \mathbf{1}_{H} \quad \text { or } \\
& M_{z}=M_{z}(T)=M(T) H_{z} \quad \text { so that using Eq. (8b), } \\
& H_{z}[1+M(T)]=\sin \phi .
\end{aligned}
$$

Using the fundamental equation (3), we have along the boundaries $z=0,1$,

$$
\begin{aligned}
& H^{2}=H_{x}^{2}+H_{z}^{2} \approx\left[1-2 M(T) \sin ^{2} \phi\right] \text { or } \\
& H \approx 1-M(T) \sin ^{2} \phi .
\end{aligned}
$$

The last equation leads us to solve $H^{2}-H+M(T) \sin ^{2} \phi=0$ in the variable $H$. One of the roots in the high field approximation (3) is $H \approx M(T) \sin ^{2} \phi \ll 1$. The only physical solution is

$$
H \approx 1-M(T) \sin ^{2} \phi=1-m \sin ^{2} \phi,
$$

where due to (3), $m=O\left(\epsilon_{H}\right)$. Using in (14e) Eqs. (4a) and (10b) along the boundaries $z=0,1$, we obtain for the dimensionless magnetic field $H$ calculated along the boundaries, two linear functions of the variable $x$. Identifying the coefficients, we have

$$
\alpha_{1}=-\epsilon_{H} \sin ^{2} \phi
$$

and

$$
h(0)=h(1)=1-\sin ^{2} \phi\left\{M\left(T_{0}\right)-\epsilon_{H}\left[T_{\text {ref }}-T_{0}\right]\right\} \approx O(1) .
$$

Thus we can define $m=\epsilon_{H} m_{1}=\left\{M\left(T_{0}\right)-\epsilon_{H}\left[T_{\text {ref }}-T_{0}\right]\right\}$, so that $m_{1}=O(1)$.

\section{B. Across the layer $0 \leq z \leq 1$}

Equation (14f) defines the value of $\alpha_{1}$ across the whole layer, since we know it to be a constant. Furthermore, from Eqs. (11), (14a), and (14g), the norm of the magnetic field $\sqrt{H_{x}^{2}+H_{z}^{2}}=h+\alpha_{1} x$ can be rewritten as

$$
h(z)+\alpha_{1} x=\sqrt{[\cos \phi+g(z)]^{2}+\left[h_{z}(z)+\frac{d g}{d z} x\right]^{2}} .
$$

Since from (14f) we know $\alpha_{1}$ to be of $O\left(\epsilon_{H}\right)$, the dependence on $x$ of the norm of the magnetic field $H$ should be very weak. Accordingly, the terms on the RHS of the last equation (15a) multiplying $x$ should also be very small. Thus, we can expand the RHS in terms of a power series in $x$, which yields

$$
\begin{aligned}
h(z)+\alpha_{1} x \approx & \sqrt{[\cos \phi+g(z)]^{2}+\left[h_{z}(z)\right]^{2}} \\
& \times\left[\begin{array}{c}
h_{z}(z) \frac{d g}{d z} x \\
1+\frac{1}{[\cos \phi+g(z)]^{2}+\left[h_{z}(z)\right]^{2}}
\end{array}\right]
\end{aligned}
$$

neglecting the terms in $x^{2}$. This leads to

$$
\begin{aligned}
& h(z)=\sqrt{[\cos \phi+g(z)]^{2}+h_{z}(z)^{2}} \text { and } \\
& \alpha_{1}=\frac{h_{z}(z) \frac{d g}{d z}}{\sqrt{[\cos \phi+g(z)]^{2}+\left[h_{z}(z)\right]^{2}}} .
\end{aligned}
$$

Then, identifying last equation (15c), to (14f), we obtain

$$
\frac{\frac{d g_{1}}{d z} h_{z}(z)}{\sqrt{[\cos \phi+g(z)]^{2}+\left[h_{z}(z)\right]^{2}}}=-\sin ^{2} \phi .
$$

Hence $\left(d g_{1} / d z\right) h_{z}(z)=-\sin ^{2} \phi+O\left(\epsilon_{H}\right)$. 
Looking back at the second Maxwell equation (5b) and using (10a), we obtain the dimensionless version of the induction hypothesis $(5 b)$ as

$$
\frac{\partial H_{x}}{\partial x}+\frac{\partial H_{z}}{\partial z}=-\epsilon_{H} \frac{\left[H_{x}+H_{z} \frac{d t}{d z}\right]}{H},
$$

that becomes using (10b), (11), and (14a),

$$
\begin{aligned}
\frac{\partial H_{z}}{\partial z} & =\frac{d^{2} g}{d z^{2}} x+\frac{d h_{z}}{d z} \\
& =-\epsilon_{H}\left[h_{x}(z)+\frac{d t}{d z}\left(h_{z}(z)+\epsilon_{H} \frac{d g_{1}(z)}{d z} x\right)\right] /\left[h+\alpha_{1} x\right] .
\end{aligned}
$$

Keeping only terms up to $O\left(\epsilon_{H}\right)$, we eliminate the $x$ dependency and obtain from the last equation (15f)

$$
\frac{d h_{z}(z)}{d z}=-\epsilon_{h} \frac{\cos \phi+\frac{d t}{d z} h_{z}(z)}{\sqrt{\cos ^{2} \phi+h_{z}^{2}(z)}}+O\left(\epsilon_{H}^{2}\right) .
$$

Introducing $(15 \mathrm{~g})$ in $(15 \mathrm{c})$ shows us also that $\frac{d h}{d z}$ is $O\left(\epsilon_{H}\right)$.

Let us introduce in the last equation $(15 \mathrm{~g})$, the power expansion (13) in $\epsilon_{H}$. One has

$$
\begin{aligned}
& \frac{d h_{0}}{d z}=\frac{d h_{0 z}}{d z}=0 \quad \text { and } \\
& \frac{d h_{1 z}}{d z}=-\left[\cos \phi+\frac{d t_{0}}{d z} \sin \phi\right] .
\end{aligned}
$$

The zero order term $h_{0}(z)$ and $h_{0 z}(z)$ are thus constant. From $(8 \mathrm{~b})$ and $(14 \mathrm{~g})$ defining the boundary values of the magnetic field, one obtains immediately

$$
\begin{aligned}
& h(z)=1+\epsilon_{H} h_{1}(z), \quad h_{z}(z)=\sin \phi+\epsilon_{H} h_{z 1}(z), \\
& \frac{d g_{1}}{d z}=-\sin \phi+O\left(\epsilon_{H}\right),
\end{aligned}
$$

using also (15d). Thus looking back at the Maxwell equations (5b) and (15h), we obtain the dimensionless version of the induction hypothesis as

$$
\frac{d h_{z}(z)}{d z} \approx-\epsilon_{H}\left[\cos \phi+\frac{d t}{d z} \sin \phi\right]+O\left(\epsilon^{2}\right) .
$$

Now let us calculate from (15c), $d h / d z$ $=(d / d z) \sqrt{\left[\cos \phi+\epsilon_{H} g_{1}(z)\right]^{2}+h_{z}(z)^{2}}$, so that

$$
\frac{d h}{d z}=\frac{\epsilon_{H} \cos \phi \frac{d g_{1}}{d z}+h_{z} \frac{d h_{z}}{d z}}{h} .
$$

Using the asymptotic result (15i) and (15j), we finally get

$$
\frac{d h}{d z}=-\epsilon_{H}\left[2 \sin \phi \cos \phi+\sin ^{2} \phi \frac{d t}{d z}\right] .
$$

\section{THE VELOCITY AND TEMPERATURE SOLUTIONS}

We can summarize all previous results

$$
\frac{\partial H}{\partial x}=-\epsilon_{H} \sin ^{2} \phi \quad \text { and } \quad \frac{\partial H}{\partial z}=\frac{d h}{d z} .
$$

Using (16), the dimensionless equivalent of the momentum balance (6d) reads

$$
\frac{d^{3} u}{d z^{3}}+\mathrm{Ra}-\frac{\mathrm{Ra}_{m}}{\epsilon_{H}}\left[\frac{d h}{d z}+\epsilon_{H} \sin ^{2} \phi \frac{d t}{d z}\right]=0 .
$$

The adimensionalization process introduces two positive dimensionless numbers $\mathrm{Ra}$ and $\mathrm{Ra}_{m}$ which are proportional to $\beta$ :

(1) The number $\mathrm{Ra}=\rho_{0} \alpha g \beta d^{4} / \eta \kappa$ is the classical Rayleigh number. ${ }^{1,2,4,7,8}$

(2) $\mathrm{Ra}_{m} / \epsilon_{H}$, where the numerator is the usual magnetic Rayleigh number $\operatorname{Ra}_{m}=\beta^{2} \mu_{0} K^{2} d^{4} / \eta \kappa$ (Refs. 8 and 10) and the denominator is the quantity $\epsilon_{H}=K \beta d / H^{e} \ll 1$.

Thus, using (151) to express $\frac{d h}{d z}$ in the RHS of the momentum balance equation (17), this last one finally becomes

$$
\frac{d^{3} u}{d z^{3}}+\mathrm{Ra}\left[1+\frac{\mathrm{Ra}_{m}}{\mathrm{Ra}} \sin 2 \phi\right]=\frac{d^{3} u}{d z^{3}}+\operatorname{Ra} \mathcal{H}(\phi)=0 .
$$

We are introducing herewith the factor

$$
\mathcal{H}(\phi)=1+\frac{\mathrm{Ra}_{m}}{\mathrm{Ra}} \sin 2 \phi .
$$

Equation (18a) is clearly the momentum balance equation studied by Birikh ${ }^{2}$ up to a small transformation. Indeed Birikh studied

$$
\frac{d^{3} u}{d z^{3}}+\mathrm{Ra}=0
$$

All that we have to do is to multiply his solution by the factor $\mathcal{H}(\phi)$.

The energy balance equation ( $7 b$ ) becomes equivalent to the usual Fourier equation. ${ }^{5,8}$ Indeed, the advective term due to the horizontal component of the gradient of the magnetic field gives a contribution proportional to $\alpha_{1} \times O\left(\epsilon_{H}\right)$ and is thus negligible. We thus have explicitly

$$
\frac{d^{2} t}{d z^{2}}-u(z)=0 .
$$

The solutions for $u(z)$ and $t(z)$ obtained from Eqs. (18a) and (18d) are closed by the dimensionless expressions of the boundary conditions along the rigid plates

$$
t=u=0, \quad \text { at } z=0,1
$$

and by the dimensionless form of the return flow assumption (6b), 


$$
\int_{0}^{1} u(z) d z=0
$$

We have thus five conditions to solve our problem at zero order in $\epsilon_{H}$. Its solution is unique: the reference velocity $u$ is given by

$$
u(z)=-\frac{\operatorname{Ra} \mathcal{H}}{12} z(z-1)(2 z-1),
$$

whose extrema are $\pm \operatorname{Ra} \mathcal{H} / 72 \sqrt{3}$, at $z=1 / 2 \pm \sqrt{1 / 12}$. The velocity reaches a maximal value $\operatorname{Max}(u)=\operatorname{Ra} \mathcal{H} / 72 \sqrt{3}$ $\approx 0.008 \mathrm{Ra} \mathcal{H}$ at $z_{\max }=0.788$. Using explicitly the velocity profile (19) in the RHS of the energy equation (18d), we obtain the temperature profile

$$
t(z)=-\frac{\operatorname{Ra} \mathcal{H}}{720} z\left(6 z^{4}-15 z^{3}+10 z^{2}-1\right) .
$$

\section{A. The classical case of Birikh (Refs. 2-4) or $\mathcal{H}=1$}

When $\mathcal{H}=1$, we are considering three possible cases. Either there is no magnetic field present so that $\mathrm{Ra}_{m}=0$ or the magnetic field is present but it is parallel or perpendicular to the rigid boundaries. In all three cases, the profiles $u(z), t(z)$ of velocity and temperature will thus be identical to the ones for a ferrofluid in a lateral temperature gradient when there is no magnetic field present. The specific properties of the ferrofluid will not intervene, in this reference situation. The solutions are thus the classical solutions $u_{\mathrm{BKD}}$, given by

$$
u_{\mathrm{BKD}}(z)=-\frac{\mathrm{Ra}}{12} z(z-1)(2 z-1),
$$

and the temperature profile $t_{\mathrm{BKD}}$

$$
t_{\mathrm{BKD}}(z)=-\frac{\mathrm{Ra}}{720} z\left(6 z^{4}-15 z^{3}+10 z^{2}-1\right) .
$$

One can identify very easily these last expressions (21a) and (21b) with the original solutions of Birikh, ${ }^{2}$ Kirdyashkin, ${ }^{3}$ and Davis, ${ }^{4}$ provided an appropriate rescaling.

\section{B. On an apparent contradiction}

Using (151) and (20), the magnetic field is given by

$$
h(z)=1-\epsilon_{H}\left[z \sin 2 \phi+t(z) \sin ^{2} \phi+C\right] .
$$

Since $h(0)=1-\epsilon_{H} m_{1} \sin ^{2} \phi$ and $t(0)=0$, see $(14 \mathrm{~g})$ and $(18 \mathrm{e})$, we have the value of $C$

$$
C=\sin ^{2} \phi m_{1} .
$$

Unhappily, we cannot satisfy that with the value of $C$, the other boundary condition at $z=1$. We end up with $h_{1}(0)$ $\neq h_{1}(1)$. This is not a contradiction with the boundary conditions deduced from $(8 \mathrm{~b})$ that lead to $(14 \mathrm{~g})$; we can get correctly neither $h_{1}(z)$ nor $h_{1 z}$ from the previous derivation.

\begin{tabular}{|c|c|c|c|}
\hline Symbol & Value & $\begin{array}{l}\text { SI or } \\
\text { CGS }\end{array}$ & Quantity \\
\hline $\bar{\rho}$ & 1530 & $\mathrm{~kg} / \mathrm{m}^{3}$ & Density \\
\hline$\nu$ & $6.5 \times 10^{-6}$ & $\mathrm{~m}^{2} / \mathrm{s}$ & Kinematic viscosity \\
\hline$\kappa$ & $8.2 \times 10^{-8}$ & $\mathrm{~m}^{2} / \mathrm{s}$ & Heat diffusivity \\
\hline$\alpha$ & $6.0 \times 10^{-4}$ & $\mathrm{~K}^{-1}$ & Thermal expansion \\
\hline$B_{\text {sat }}$ & $60.0 \times 10^{-3}$ & Tesla & $\begin{array}{l}\text { Magnetic induction } \\
\text { at saturation }\end{array}$ \\
\hline$M_{\text {sat }}$ & $4.8 \times 10^{4}$ & $\mathrm{~A} / \mathrm{m}$ & Saturation magnetization \\
\hline$K$ & 29 & $\mathrm{~A} / \mathrm{K}$ & Pyromagnetic coefficient \\
\hline$H^{e}$ & $\gg 4.8 \times 10^{4}$ & $\mathrm{~A} / \mathrm{m}$ & Strong external field \\
\hline$d$ & $1-10$ & $\mathrm{~mm}$ & Variable width \\
\hline$\beta$ & $0.1-1.0$ & $\mathrm{~K} / \mathrm{mm}$ & Variable lateral gradient \\
\hline$g$ & $9.81 \times 10^{-n}$ & $\mathrm{~ms}^{-2}$ & $\begin{array}{l}\text { Variable gravity level, } \\
6 \geq n \geq 0\end{array}$ \\
\hline $\mathrm{Ra}$ & $11 \times 10^{-n} d^{4} \beta$ & & $\begin{array}{l}\text { Thermal Rayleigh } \\
\text { number }\end{array}$ \\
\hline $\mathrm{Ra}_{m}$ & $1.28 d^{4} \beta^{2}$ & & $\begin{array}{l}\text { Magnetic Rayleigh } \\
\text { number }\end{array}$ \\
\hline$\epsilon_{H}$ & $29 d \beta / H^{e}$ & & $\begin{array}{l}\text { Coupling between magnetic } \\
\text { field and temperature }\end{array}$ \\
\hline $\mathrm{Ra}_{m} / \mathrm{Ra}$ & $0.116 \times 10^{n} \beta$ & & \\
\hline
\end{tabular}
Up to the first order in $\epsilon_{H}$, this is logical since in (17), the factor $\mathrm{Ra}_{m} / \epsilon_{H}$ requires us to introduce $d h_{1} / d z$, proportional to $\epsilon_{H}$. This enables us to get rid of the $\epsilon_{H}$ dependency in (17) and get the zero order differential equation (18a). These last
TABLE I. Fluid properties for EMG 901 (Ref. 17) and the dimensionless numbers.

equations (18a) and (18d) do not depend on the magnetic field explicitly and constitutes a linear system of the fifth order with the four boundary conditions at $z=0, z=1$, and the fifth one, (18f) closing the system. In the approximation scheme developed here, we do not use any of the boundary conditions linked to the magnetic field.

Beyond the zeroth order term of the expansion in $\epsilon_{H}$, the dependence in $z$ is much more complicated. The calculations express that the magnetic field exhibits a very small correction across the layer which "disturbs" the original $t, u$ profiles. To solve the real problem, one must introduce a $2 \mathrm{D}$ model for $u, t, h$. Nevertheless, the error which we are leaving is $O\left(\epsilon_{H}\right)$.

\section{EXPERIMENTAL VALUES OF THE DIMENSIONLESS PARAMETERS}

Before discussing the theoretical results of the present model, we will consider its physical relevance. We consider the physical data that define a well known ferrofluid, EMG 901 (Ref. 17) (see Table I) as well as the validity range of our problem.

We suppose a layer of infinite lateral extent. In practice, it means that the layer total length is large enough to have a middle region far enough from the lateral walls, where their influence can be neglected. ${ }^{2-4}$ If $L$ is the distance between both lateral walls, we must assume $d \ll L$. Another limitation to take into account is that the total drop of temperature, from one vertical end wall to the other, does not include a phase change. The total temperature drop from the hot wall to the cold one defines $\beta$, since $T_{\text {hot }}-T_{\text {cold }} \approx \beta L$. A reasonable value on $\beta$ for EMG 901 means to know the temperature of the liquid-gas phase change and of the solid-liquid phase change. That liquid is an isoparaffin solvent, whose boiling 
point is around $250{ }^{\circ} \mathrm{C}$. EMG 901 belongs to the alkylate family. Since the freezing point of naphtha is $-54{ }^{\circ} \mathrm{C}$, we assume EMG 901 to be a liquid, at normal temperature. Measuring $d$ in $\mathrm{mm}$ and $\beta$ in $\mathrm{K} / \mathrm{mm}$ and assuming $L=15 \mathrm{~cm}, \beta$ should be lower than $1 \mathrm{~K} / \mathrm{mm}$ so that the total temperature drop should be less than $150{ }^{\circ} \mathrm{C}$. We will thus consider $0.1 \leq \beta \leq 1$.

We use the data gathered in Table I to estimate the following numbers. One notes that $\mathrm{Ra}_{m}$ varies from $1.28 \times 10^{4}$ for $d=10$ and $\beta=1$ down to 0.0128 for $d=1$ and $\beta=0.1$ while $\mathrm{Ra}$ varies between its maximum value $11 \times 10^{4}$ on Earth at $d=10$ and $\beta=1$, down to its minimum $\mathrm{Ra}=1.1 \times 10^{-6}$ in microgravity $(n=6)$ at $d=1.0$ and $\beta=0.1$. The ratio of the parameters $\mathrm{Ra}_{m} / \mathrm{Ra}$ does not depend on the depth $d$. On Earth, at $\beta=1, \mathrm{Ra}_{m} / \mathrm{Ra}$ reaches a maximum value $0.116<1$. In microgravity, for $\beta=0.1$, the ratio $\mathrm{Ra}_{m} / \mathrm{Ra}$ is larger than 1 . It varies from 1.16 at $n=2$ up to 11600 at $n=6$. The quantity $\mathrm{Ra}_{m} / \mathrm{Ra}$ thus covers a very large range of physical values and depends highly on the gravity level. From the explicit definition of $\mathrm{Ra}_{m} / \mathrm{Ra} \approx 0.116 \times 10^{n} \beta$, one can define a gravity level $n>0$ and $\beta_{n} \approx 8.62 \times 10^{-n}$, a critical value of the lateral temperature gradient $\beta$ such that $\mathrm{Ra}_{m} / \mathrm{Ra}=1$. For lower gravity level (meaning thus larger $n$ ) or for larger value of $\beta$ $>\beta_{n}, \mathrm{Ra}_{m}>\mathrm{Ra}$. For EMG 901, the magnetization at saturation is $M_{\text {sat }}=4.8 \times 10^{4} \mathrm{~A} / \mathrm{m}^{17}$ Our study is valid only in the framework of a strong magnetic field (4a), so that we must consider a magnetic field larger than $M_{\text {sat }}$ to obey the strong field assumption. Then, should we take an exterior magnetic field larger than $4.8 \times 10^{4} \mathrm{~A} / \mathrm{m}$, our choice of parameters (see Table I) shows $\epsilon_{H}$ to be less than 0.001. It is thus logical to consider that

$$
\epsilon_{H} \ll 1 \text {. }
$$

The last inequality which we used to solve the inclined magnetic field problem is now experimentally substantiated.

\section{THE PROFILES OF VELOCITY AND TEMPERATURE FOR AN INCLINED MAGNETIC FIELD}

In the case of an inclined strong magnetic field, the velocity and temperature profiles (19) and (20) can be written as

$$
\begin{aligned}
& u(z)=\mathcal{H}(\phi) u_{\mathrm{BDK}}(z), \\
& t(z)=\mathcal{H}(\phi) t_{\mathrm{BDK}}(z) .
\end{aligned}
$$

The whole discussion reduces to the physical and mathematical meaning of the factor $\mathcal{H}(\phi)$ which varies with the obliquity and combines the gravity level, the saturation magnetization and the temperature gradient. This opens new possibilities which should be carefully discussed as these suggest future experiments. This factor dilates the Birikh shape when $\sin 2 \phi$ is positive or compresses it when $\sin 2 \phi$ is negative. For a given inclination $\phi$, the maximum of the velocity (25) is $\left[\mathrm{Ra}+\mathrm{Ra}_{m} \sin 2 \phi\right] / 72 \sqrt{3}$. This last function varies with the inclination angle $\phi$. It reaches its maximum value $\left[\mathrm{Ra}+\mathrm{Ra}_{m}\right] /(72 \sqrt{3})$ at $\phi=\pi / 4$, and its minimum value $\left[\mathrm{Ra}-\mathrm{Ra}_{m}\right] /(72 \sqrt{3})$ at $\phi=3 \pi / 4$.
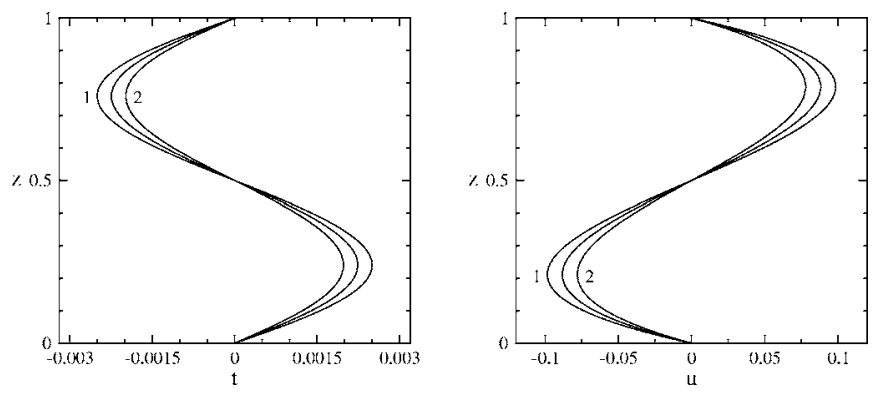

FIG. 2. Temperature and velocity on Earth. $d=1 \mathrm{~mm}, \beta=1 \mathrm{~K} \mathrm{~mm}^{-1}$, Ra $=11, \operatorname{Ra}_{m}=1.28$. Curve 1: $\phi=45^{\circ}$. Middle curve: $\phi=0^{\circ}$. Curve 2: $\phi=135^{\circ}$.

Equation (25) has a very important consequence. Indeed, the velocity can be equal to zero and the temperature gives a pure conductive solution for a critical angle $\phi=\phi_{\text {crit }}$ whenever $\mathcal{H}\left(\phi_{\text {crit }}\right)=1+\left(\mathrm{Ra}_{m} / \mathrm{Ra}\right) \sin 2 \phi_{\text {crit }}=0$. We shall now consider two cases depending on the ratio $\mathrm{Ra}_{m} / \mathrm{Ra}$ using the discussion of Sec. VI for realistic conditions since $\mathrm{Ra}_{m} / \mathrm{Ra}$ $<1$ and $\mathrm{Ra}_{m} / \mathrm{Ra}>1$ corresponds to a microgravity situation.

(i) Case $\mathrm{Ra}>\mathrm{Ra}_{m}$. Then, whatever the value of $\sin 2 \phi$, $\mathcal{H}$ is always positive. $\mathcal{H}$ is larger than 1 when $\phi$ belongs to the first or the third quadrant, and less than 1 , when $\phi$ belongs to the second or the fourth quadrant. Using Table I, we are showing in Fig. 2, the results for three inclinations, at $\mathrm{Ra}_{m} / \mathrm{Ra} \approx 0.116$. For $d=1, \beta=1, \mathrm{Ra}=11, \mathrm{Ra}_{m}=1.28$, the inclination can compress or dilate the Birikh profile very slightly, given by the middle curve (see Fig. 2). This follows from the comparison of the profiles of $u(z)$ and $t(z)$ obtained using (21a) and (21b) in the absence of a magnetic field, to the ones given by (25), for $\phi=45^{\circ}, 135^{\circ}$ (curves 1 and 2, respectively).

(ii) Case $\mathrm{Ra} \leq \mathrm{Ra}_{m}$. Then, when $\phi$ belongs to the first or the third quadrant, $\mathcal{H}$ is positive and larger than 1 . The shape of the profiles of velocity and temperature do not differ much from the previous case. But, when $\mathrm{Ra} \leq \mathrm{Ra}_{m}$, the quantity $\mathcal{H}$ can also be negative or even equal to zero. Indeed, if $\sin \phi \cos \phi$ is negative, i.e., when $\phi$ belongs to the second or the fourth quadrant, the case $\mathcal{H}=0$ cannot be excluded anymore. It means to solve $\sin \phi \cos \phi=-\mathrm{Ra} /\left(2 \mathrm{Ra}_{m}\right)$. Let us call, $y$ the square of either $\sin \phi$ or $\cos \phi$ so that one can write $\sin \phi \cos \phi= \pm \sqrt{y(1-y)}$. Then, $\mathcal{H}=0$ becomes equivalent to $y^{2}-y+\mathrm{Ra}^{2} / 4 \mathrm{Ra}_{m}^{2}=0$. The roots of the last equation are given by

$$
y_{ \pm}=\frac{1}{2}\left[1 \pm \sqrt{1-\frac{\mathrm{Ra}^{2}}{\mathrm{Ra}_{m}^{2}}}\right] \text { with } y_{+}+y_{-}=1 \text {. }
$$

Thus to be physically meaningful, both roots $y_{ \pm}$must be real and positive quantities, less than 1 . The following condition should necessarily be satisfied: $\mathrm{Ra}_{m} / \mathrm{Ra} \geq 1$. That condition is, by definition independent upon the depth $d$. Only if it is fulfilled, will we have $\mathcal{H}<0$, for $y_{-}<y<y_{+}$. Indeed, for $y$ $>y_{+}$or $y<y_{-}, \mathcal{H}$ is always positive. But, for all $y$ such that $y_{-} \leq y \leq y_{+}$, one has 

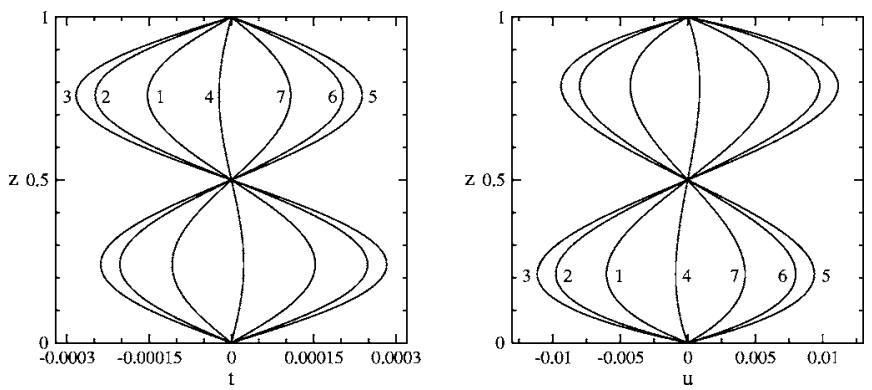

FIG. 3. Temperature and velocity in low gravity $(n=2) . d=1 \mathrm{~mm}, \beta$ $=1 \mathrm{~K} \mathrm{~mm}^{-1}, \mathrm{Ra}=0.11, \mathrm{Ra}_{m}=1.28$. Curve 1: $\phi=15^{\circ}$. Curve 2: $\phi=30^{\circ}$. Curve 3: $\phi=45^{\circ}$. Curve 4: $\phi=90^{\circ}$. Curve 5: $\phi=135^{\circ}$. Curve 6: $\phi=150^{\circ}$. Curve 7: $\phi=165^{\circ}$.

$$
\operatorname{Abs}[\sin \phi \cos \phi]=\sqrt{y(1-y)}>\frac{\mathrm{Ra}}{2 \mathrm{Ra}_{m}}
$$

and thus $\mathcal{H}<0$ since $\sin \phi \cos \phi<0$.

The above discussion is quite easy to illustrate, at least theoretically. Indeed, for a given ferrofluid like EMG901 (see Table I) at a given magnetic field and lateral gradient $\beta$, the only freewheeling parameter left in $\mathrm{Ra} / \mathrm{Ra}_{m}$ is the gravity level $n$. In microgravity $n=2$ at $\beta=1 \mathrm{~K} \mathrm{~mm}^{-1}$, the ratio of $\mathrm{Ra}_{m} / \mathrm{Ra} \approx 0.116 \times 10^{n} \beta$ is now $\approx 11.6$, which is larger than one and we obtain the velocity and temperature profiles shown in Fig. 3.

A new possibility appears since $\mathcal{H}=0$ for a certain value of $y$ given by $y_{+}$or $y_{-}$, then $u(z)=t(z)=0$ for all $z$. The zero order solution is a conductive one and there is no flow. The temperature profile reduces to $T=T^{\mathrm{ref}}-\beta d x$ across the whole layer. In other words the solution is motionless and conductive when $2 \phi_{\text {cr }}=\operatorname{arc} \sin \left\{-\mathrm{Ra} / \mathrm{Ra}_{m}\right\}$. Thus $2 \phi_{\mathrm{cr}} \approx-\mathrm{Ra} / \mathrm{Ra}_{m}$ $+\frac{1}{6}\left[\mathrm{Ra} / \mathrm{Ra}_{m}\right]^{3}-\frac{3}{40}\left[\mathrm{Ra} / \mathrm{Ra}_{m}\right]^{5}$. When one obtains a precise value of $y_{+}$, this defines a certain angle $\phi_{\mathrm{cr}}=\pi / 2+\theta_{\mathrm{cr}}$ in the second quadrant. But $1=y_{+}+y_{-}$intervenes also, to calculate the other solution of (26). Thus the velocity will become zero also for another angle in the second quadrant, given by $\pi-\theta_{\text {cr }}$ using classical trigonometry properties. The same reasoning will apply in the fourth quadrant. Physically, at zero order in $\epsilon_{H}$, the lateral pressure gradient induced by the temperature is exactly compensated by the inclined magnetic field. This solution is a very unexpected one, since it is a specific consequence of having an inclined external field. In the case of Fig. 3, for $n=2$ and $d=1 \mathrm{~mm}, \theta_{\mathrm{cr}} \approx 2.45^{\circ}$. The following scenario then applies for microgravity conditions:
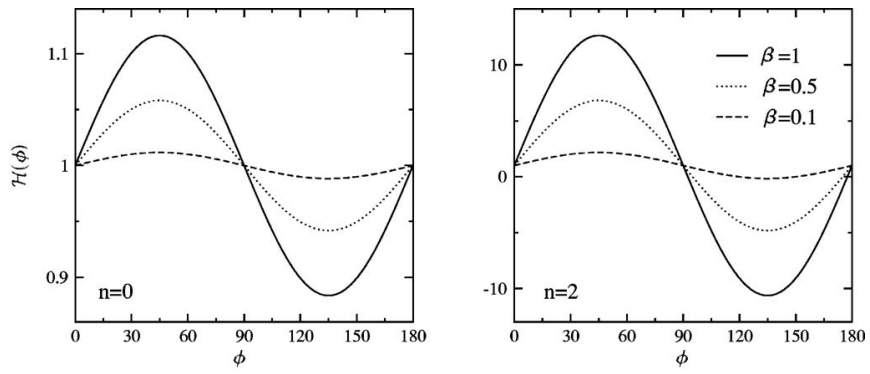

FIG. 4. The function $\mathcal{H}(\phi)$, versus $\phi$ for three different $\beta$ measured in $\mathrm{K} /$ $\mathrm{mm}$ (see legend). On Earth $(n=0)$ and in microgravity $(n=2)$.

the maxima of velocity and temperature increase first with the inclination angle $\phi$ up to a certain angle and then decrease rapidly toward zero at $\phi_{\mathrm{cr}} \approx 92.45^{\circ}$. The greatest value of the temperature (respectively of the velocity) is for a $z$ in the lower (respectively upper) half of the $[0,1]$ interval. Above that critical angle and up to $\phi=177.15^{\circ}$, the convection sense is inverted as predicted above. The greatest values of the temperature and the velocity lie now in the other half of the $[0,1]$ interval.

When $\mathcal{H}$ is negative, we have again a convective solution. But this time the convective motion is in the opposite direction of the original classical Birikh one (see Fig. 3, curves 5-7), the lateral temperature gradient induces a pressure push through gravity which is opposed to another pressure gradient due to the Kelvin force. This last one is sufficiently strong to overcome the gravitational force. The return flow has changed from direction. From the values of Table I, it follows that, this cannot be observed on Earth but could be easily reached for a microgravity environment with $n \geq 2$, as illustrated by comparing Figs. 2 and 3.

Figures 4 and 5 are other ways to illustrate the present argument, by studying directly $\mathcal{H}(\phi)$ for various lateral temperature gradients $\beta$ on Earth $n=0$ and in microgravity $n=2$, for EMG 901 (see Table I). For that ferrofluid, the ratio $\operatorname{Max}(u) / \operatorname{Max}\left(u_{\mathrm{BKD}}\right)=\mathcal{H}(\phi)$ is equal to $1+0.11610^{n} \beta \sin 2 \phi$, where $n$ is the microgravity level and $\beta$ is the temperature gradient measured in $\mathrm{K} \mathrm{mm}^{-1}$. Different behaviors can be observed.

(a) On Earth $n=0$. The velocity change, introduced by the strong inclined field, is not very large with respect to $\operatorname{Max}\left(u_{\mathrm{BDK}}\right)$ [see Figs. 4 and 5 (left)]. The magnetic field acts always in the same manner. At first the maximum velocity increases from $\operatorname{Max}\left(u_{\mathrm{BDK}}\right)$ at $\phi=0^{\circ}$ with
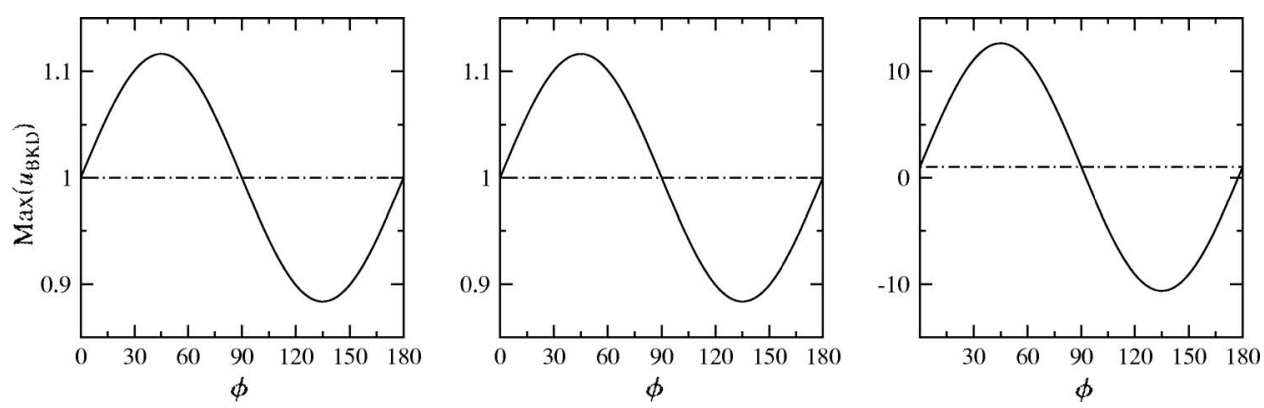

FIG. 5. In the ordinate, the maximum of the velocity scaled by $\operatorname{Max}\left(u_{\mathrm{BKD}}\right)$ versus $\phi$ for $\epsilon_{H}=0.0001$. Left: $\mathrm{Ra}=6875, \mathrm{Ra}_{m}=800$. Center: $\mathrm{Ra}=11$, $\mathrm{Ra}_{m}=1.28$. Right: $\mathrm{Ra}=0.11, \quad \mathrm{Ra}_{m}$ $=1.28$. 

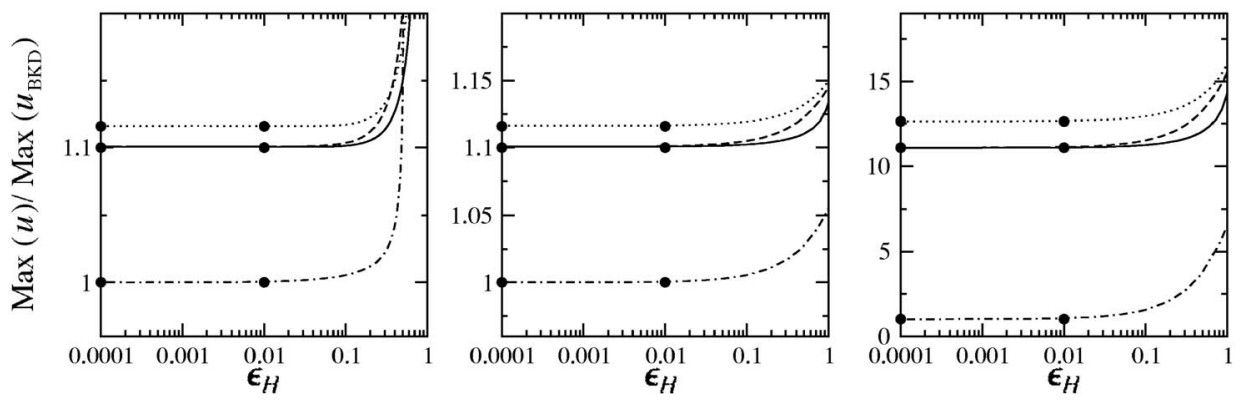

FIG. 6. In the ordinate, $\operatorname{Max}(u) / \operatorname{Max}\left(u_{\mathrm{BKD}}\right)$ as a function of $\epsilon_{H}$, for various inclinations $\phi, \phi=30^{\circ}$, $\phi=60^{\circ}, \phi=45^{\circ}, \phi=90^{\circ}$. Left: Ra $=6875, \mathrm{Ra}_{m}=800$. Center: $\mathrm{Ra}=11$, $\mathrm{Ra}_{m}=1.28$. Right: $\mathrm{Ra}=0.11, \quad \mathrm{Ra}_{m}$ $=1.28$. the angle of inclination and reaches a maxima value, which is slightly above the Birikh maximum $\operatorname{Max}\left(u_{\mathrm{BDK}}\right)$, at a finite angle of inclination. Beyond that value, the maximum of the velocity decreases from that maximal value, becoming equal to $\operatorname{Max}\left(u_{\mathrm{BDK}}\right)$ for an inclination very near to $\phi \approx \pi / 2$, then continues down to its minimal value somewhere around $\phi \approx 3 \pi / 4$. Past that angle, the velocity increases again towards $\operatorname{Max}\left(u_{\mathrm{BDK}}\right)$ at $\phi=\pi$.

(b) One way to decrease $\mathrm{Ra}$ and $\mathrm{Ra}_{m}$, simultaneously, is achieved by using smaller values of $d$ or $\beta$, since these quantities appear, with a positive exponent, in the definitions of $\mathrm{Ra}$ and $\mathrm{Ra}_{m}$ (see Table I). However, reducing $d$ only at a given $\beta$ keeps the same value of the ratio $\mathrm{Ra}_{m} / \mathrm{Ra}$. (Compare Fig. 5 left and center.)

(c) Another way to reduce $\mathrm{Ra}$, while keeping the same value of $\mathrm{Ra}_{m}$, is to vary the gravity level as shown in Figs. 4 and 5. In the case of EMG 901, we considered a microgravity case at $n=2$, which is equivalent to reducing 100 times the gravity level, so that Ra vary now like $0.11 d^{4} \beta$ while $\operatorname{Ra}_{m}=1.28 d^{4} \beta^{2}$ and the ratio $\mathrm{Ra}_{m} / \mathrm{Ra} \approx 11.54 \beta$ varies linearly with the temperature gradient, whatever the depth $d$. While the shape of the curve seems identical, we observe a very physical different behavior than in the previous case since a gradient of temperature $0.1<\beta<1.0$ is sufficient to have $\mathrm{Ra}_{m} / \mathrm{Ra}>1$. One observes that $\mathcal{H}(\phi)$ can now become equal to zero for two peculiar values of $\phi$. The curves at $\beta=0.5,1.0$ in Fig. 4 give a conductive profile at $\phi_{\mathrm{cr}}>90^{\circ}$ and $\phi_{\mathrm{cr}}<\pi$. Between those values of $\phi, \mathcal{H}$ is negative: the profile of velocity has changed direction (see Fig. 4).

\section{VALIDATION RANGE OF THE STRONG FIELD APPROXIMATION}

Figures 4 and 5 contain the same kind of information. However Fig. 4 illustrates the analytical solution given by (25), whereas Fig. 5 refers to direct numerical simulations. These last are obtained from the governing system of equations $(17),(15 \mathrm{~g}),(15 \mathrm{k})$, and $(18 \mathrm{~d})$ and the boundary conditions $(14 \mathrm{~g}),(18 \mathrm{e})$, and (18f) using the continuation software AUTO97 (Ref. 18) and computed in the space of parameters defined by Table I. Furthermore, in the rest of the paragraph, the maximum velocity (in absolute value), $\operatorname{Max}(u)$, is scaled by $\operatorname{Max}\left(u_{\mathrm{BKD}}\right)=\mathrm{Ra} /(72 \sqrt{3})$. This last is the maximum velocity of the Birikh solution [see (21a)]. ${ }^{2-4}$
Hence, when the magnetic field is absent, horizontal or vertical, the maximum velocity is always equal to $\operatorname{Max}\left(u_{\mathrm{BKD}}\right)[\mathrm{see}(21 \mathrm{a})]$, so that the ratio of the relative maxima will always be equal to 1 , i.e., for $\phi=0, \pi / 2, \pi$. The three curves of Fig. 6 show those relative maxima, at four chosen $\phi$, namely $\phi=15^{\circ}, \phi=30^{\circ}, \phi=45^{\circ}$, and $\phi=90^{\circ}$, as a function of $\epsilon_{H}$, using the data from Table I.

As long as $\epsilon_{H}$ is small enough the ratio of the velocity maxima is a constant for a given $\phi$ and is equal to $\mathcal{H}$. Figure 6 shows this to be the case for all $\epsilon_{H}<0.01$, whatever the inclination of the magnetic field. This result is interesting because it confirms that the general solutions, given by (25), and which are independent from $\epsilon_{H}$, match perfectly the exact numerical solution once the condition $\epsilon_{H} \ll 1$ is satisfied. This is not what could be anticipated from the Maxwell equation (15k). However, the zero order solutions (19), (20), and (25), which has been obtained from (18a), rests on the assumption that $\epsilon_{H}$ is a small quantity (see Sec. V). This last assumption is always true in our case, since the strong field means that $\epsilon_{H}=(K \beta d) / H^{e}$ should decrease with the increase of the external field. As the ratio of these velocities does not vary as a function of the precise value $\epsilon_{H}$ [see Fig. 6], it demonstrates a posteriori, that the zero order development of (18a) is largely sufficient and that there is no need to go over to the next expansion order. This is true for any inclined field. Figure 5 shows this to be true for all $\phi$, between $0, \pi$.

\section{CONCLUSION}

We have considered here a thin layer of a ferrofluid, heated laterally and submitted to an inclined magnetic strong field and looked for its zeroth order term in a power series expansion in terms of a small parameter, $\epsilon_{H}$ that measures the strength of the magnetization relatively to the applied magnetic field $H^{e}$. Two conclusions can be drawn:

(a) For the vertical magnetic field and for the horizontal one, the noninductive hypothesis applies even if one recovers the classical Birikh solution for a Newtonian fluid layer submitted to lateral heating.

(b) For any other inclination, we used a development (13) in a small parameter $\epsilon_{H}$ linked to the variation of magnetization in the momentum equation (17). It was giving rise to a solution (25) which is simply the product of $\mathcal{H}=1+\mathrm{Ra}_{m} / \mathrm{Ra} \sin 2 \phi$ a term depending on the thermal Rayleigh number $\mathrm{Ra}$, the magnetic Rayleigh 
number $\mathrm{Ra}_{m}$, and the inclination angle $\phi$, by the classical solution obtained by Birikh.

Using the phenomenological data provided for a typical ferrofluid EMG 901, we estimate the order of magnitude of these parameters to compare the analytical results with the numerical solution. In the strong field case, the velocity and temperature profiles given by (25) are indistinguishable from the exact numerical solution whatever the magnetic field strength, its inclination, the depth of the layer or the gravity level. This leads to the main conclusion. The profiles of velocity and temperature are now obtained from the product of two quantities. The first one is the corresponding laminar profiles for an usual Newtonian fluid submitted to a lateral temperature gradient. ${ }^{2-4}$ That classical solution is now multiplied by the factor $\mathcal{H}$, which is, by definition, independent upon the depth of the layer. When $\mathrm{Ra}_{m} / \mathrm{Ra}$ is large enough, the role of buoyancy and magnetic field is reversed. Buoyancy is a small term, with respect to the magnetic field strength which is largely dominant. The inclination of the magnetic field can give rise to a pure conductive solution at two critical angles $\phi=\pi / 2+\theta_{\text {cr }}$ and $\phi=\pi-\theta_{\text {cr }}$ for which $\mathcal{H}=0$. For any inclination between those two angles, the direction of circulation and the temperature profile are even reverted (see Figs. 3 and 4), with respect to the classical solutions (21a) and (21b) valid in the absence of a magnetic field. These new possibilities will show up under microgravity conditions.

\section{ACKNOWLEDGMENTS}

The present publication was made possible through the agreement linking the CGRI, the FNRS (Belgium) to the Bulgarian Academy of Sciences.
${ }^{1}$ S. Chandrasekhar, Hydrodynamic and Hydromagnetic Stability (Dover, New York, 1987).

${ }^{2}$ R. V. Birikh, "Thermocapillary convection in a horizontal layer," J. Appl. Mech. Tech. Phys. 3, 69 (1966).

${ }^{3}$ A. G. Kirdyashkin, "Thermo gravitational and thermo capillary flows in a horizontal layer under the conditions of a horizontal temperature gradient," Int. J. Heat Mass Transfer 7, 1205 (1984).

${ }^{4}$ S. H. Davis, "Thermocapillary instabilities," Annu. Rev. Fluid Mech. 19, 406 (1987).

${ }^{5}$ B. A. Finlayson, "Convective instabilities of ferromagnetic fluids," J. Fluid Mech. 40, 753 (1970).

${ }^{6}$ J. L. Neuringer and R. E. Rosensweig, "Ferrohydrodynamics," Phys. Fluids 7, 1927 (1964).

${ }^{7}$ V. G. Bashtovoi and B. M. Berkovski, "Thermomechanics of ferromagnetic fluids," Magn. Gidrodin. 3, 3 (1973).

${ }^{8}$ V. G. Bashtovoi, B. M. Berkovski, and A. N. Vislovich, Introduction to Thermomechanics of Magnetic Fluids (Hemisphere, New York, 1988).

${ }^{9}$ V. G. Bashtovoi and M. S. Krakov, "Stability of the flow of a thin layer of magnetic liquid," Izv. Akad. Nauk SSSR, Mekh. Zhidk. Gaza 5, 59 (1977).

${ }^{10}$ R. E. Rosensweig, Ferrohydrodynamics (Dover, New York, 1997).

${ }^{11}$ A. G. Boudouvis, J. L. Puchalla, L. E. Scriven, and R. E. Rosensweig, "Normal field instability and patterns in pools of ferrofluid," J. Magn. Magn. Mater. 65, 307 (1987).

${ }^{12}$ R. Abou, G. Néron de Surgy, and J. E. Wesfreid, "Dispersion relation in a ferrofluid layer of any thickness and viscosity in a normal magnetic field: Asymptotic regimes," J. Phys. II 7, 1159 (1997).

${ }^{13}$ E. Blums, A. Cebers, and M. M. Maiorov, Magnetic Fluids (de Gruyter, Berlin, 1997).

${ }^{14}$ E. Blums, "Some new problems of complex thermomagnetic and diffusion driven convection in magnetic fluids," J. Magn. Magn. Mater. 149, 111 (1995).

${ }^{15}$ N. V. Tabiryan and W. Luo, "Soret feedback in thermal diffusion of suspensions," Phys. Rev. E 57, 4431 (1998).

${ }^{16}$ Jie Huang, B. D. Edwards, and D. D. Gray, "Thermoconvective instability of paramagnetic fluids in a uniform magnetic field," Phys. Fluids 9, 1919 (1997).

${ }^{17}$ J. Weilepp and H. R. Brand, "Competition between the Bénard-Marangoni and the Rosensweig instability of magnetic fluids," J. Phys. II 6, 419 (1996).

${ }^{18}$ E. Doedel, A. Champneys, T. Fairfrieve, Y. Kuznetsov, B. Sandstede, and X. Wang, "AUTO 97: Continuation and bifurcation software for ordinary differential equations," Technical Report, Department of Computer Science, Concordia University, Montreal, Canada (1997). 